\title{
edoc
}

Institutional Repository of the University of Basel

University Library

Schoenbeinstrasse 18-20

CH-4056 Basel, Switzerland

http://edoc.unibas.ch/

Year: 2014

\section{Modulation of PGC-1 $\alpha$ activity as a treatment for metabolic and muscle-related diseases}

Svensson, Kristoffer and Handschin, Christoph

Posted at edoc, University of Basel

Official URL: http://edoc.unibas.ch/dok/A6288862

Originally published as:

Svensson, Kristoffer and Handschin, Christoph. (2014) Modulation of PGC-1 $\alpha$ activity as a treatment for metabolic and muscle-related diseases. Drug discovery today, Vol. 19, H. 7. S. 1024-1029.

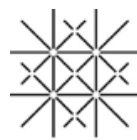


Modulation of PGC-1 $\alpha$ activity as a treatment for metabolic and muscle-related diseases

Kristoffer Svensson and Christoph Handschin*

Biozentrum, University of Basel, Klingelbergstrasse 50/70, 4056 Basel, Switzerland

Published in Drug Discov Today. 2014 19(7):1024-29. PMID: 24631683. doi:

10.1016/j.drudis.2014.02.013

Copyright (C) Elsevier; Drug Discovery Today 


\section{Reprint request}

Unfortunately, due to copyright-relatedt issues, we are not able to post the post-print pdf version of our manuscript - in some cases, not even any version of our manuscript. Thus, if you would like to request a post-production, publisher pdf reprint, please click send an email with the request to christoph-dot-handschin_at_unibas-dot-ch (see http://www.biozentrum.unibas.ch/handschin).

Information about the Open Access policy of different publishers/journals can be found on the SHERPA/ROMEO webpage: http://www.sherpa.ac.uk/romeo/

\section{Reprint Anfragen}

Aufgrund fehlender Copyright-Rechte ist es leider nicht möglich, dieses Manuskript in der finalen Version, z.T. sogar in irgendeiner Form frei zugänglich zu machen. Anfragen für Reprints per Email an christoph-dot-handschin_at_unibas-dot-ch (s. http://www.biozentrum.unibas.ch/handschin).

Informationen zur Open Access Handhabung verschiedener Verlage/Journals sind auf der SHERPA/ROMEO Webpage verfügbar: http://www.sherpa.ac.uk/romeo/ 
Modulation of PGC-1 $\alpha$ activity as a treatment for metabolic and muscle-related diseases

Kristoffer Svensson and Christoph Handschin*

Biozentrum, University of Basel, Klingelbergstrasse 50/70, 4056 Basel, Switzerland

* Corresponding author: Christoph Handschin (christoph.handschin@unibas.ch) 


\section{Abstract}

Physical inactivity is a predisposing factor for various disease states including obesity, cardiovascular disease, as well as for certain types of cancer. Regular endurance exercise mediates several beneficial effects such as increased energy expenditure and improved skeletal muscle function, and has been suggested as a therapeutic strategy for both metabolic and muscle-related disorders. "Exercise mimetic" is a collective term for compounds that can pharmacologically activate pathways which are normally induced during skeletal muscle contraction, and that could be used in the treatment of metabolic or muscle related diseases. Two such experimental "exercise mimetics" are AICAR and resveratrol, which have both been extensively studied in the context of metabolic dysfunction and muscle wasting in rodent disease models. These compounds have been postulated to activate AMP-activated protein kinase (AMPK) and sirtuin 1 (SIRT1), respectively, in skeletal muscle, and to increase the activation of the peroxisome proliferator-activated receptor $\gamma$ coactivator $1 \alpha$ (PGC-1 $\alpha$ ). PGC-1 $\alpha$ can mediate several metabolic and functional adaptations in skeletal muscle in response to physical exercise and is therefore an interesting target for the development of new "exercise mimetic" drugs. 


\section{Introduction}

While regular exercise is important to maintain health and longevity, physical inactivity predisposes for many chronic diseases of metabolic origin, such as obesity, type 2 diabetes mellitus (T2DM) and cardiovascular pathologies, as well as certain types of cancer [1]. Importantly, lack of physical activity is also a key element in the etiology of aging-associated dysfunctions, for example sarcopenia and cognitive impairment [2]. Endurance exercise has been successfully applied or suggested as a therapy in a wide array of metabolic and muscle-related disorders, for example in obese and T2DM patients [3], cancer-associated cachexia [4] and muscular dystrophies [5]. Considering the physiological impact of exercise training at the whole body level and its low cost, this strategy is a preferred choice to either surgical or pharmacological interventions in obese patients [6]. This approach however is not without inherent difficulties, since adherence to an exercise regime can be difficult to maintain, and the impairment of skeletal muscle function observed in patients suffering from diseases such as sarcopenia or muscular dystrophies can limit the use of exercise as therapy [5]. Hence, in some pathological contexts, it would be advantageous to pharmacologically activate the same pathways that are activated by muscle contraction and thus enhance the therapeutic effects of exercise. When the homeostasis of a resting muscle cell is perturbed during a contraction, several pathways are either activated or inhibited to mediate the adaptive response to exercise, both at a posttranslational and transcriptional level (reviewed in [7]). The transcriptional response in skeletal muscle to a single bout of exercise in humans can influence the expression of more than 900 genes, resulting in adaptive changes in mitochondrial metabolism, angiogenesis, $\beta$-oxidation and inflammation $[7,8]$. A key mediator of these transcriptional changes and a main point of convergence for different signaling events taking place in a contracting muscle is the peroxisome proliferatoractivated receptor $\gamma$ co-activator $1 \alpha$ (PGC-1 $1 \alpha$ ) [9]. Skeletal muscle PGC-1 $\alpha$ is increased during exercise in humans [10] and can drive a slow oxidative gene program through the activation of several transcription factors such as estrogen-related receptor $\alpha$ (ERR $\alpha$ ), nuclear respiratory factor $1 / 2$ (NRF1/2) and transcription factor $A$, mitochondrial (TFAM) [7,9]. In response to an energy stress such as muscle contraction, PGC-1 $\alpha$ can be activated by two important energy sensors: AMP-activated protein kinase (AMPK) and sirtuin 1 (SIRT1) [11] (Fig. 1). AMPK is allosterically activated by both ADP and AMP during energy deficits, and positively regulates ATP-production through phosphorylation of metabolic enzymes [12]. Interestingly, AMPK also confers long-term transcriptional adaptations in response to energy stress, by phosphorylation and activation of several transcription factors as well as transcriptional co-activators, including PGC-1 $\alpha$ [13]. Another important energy sensor during muscle contraction is SIRT1, a member of the SIRT family of class III deacetylases. SIRT1 senses perturbations in the NAD+/NADH ratio in muscle cells during exercise, and can mediate adaptive changes in mitochondrial function through deacetylation and activation of PGC- $1 \alpha$, as well as 
through direct deacetylation of several transcription factors [14]. The central role for AMPK and SIRT1 as energy sensors in skeletal muscle, as well as the presence of pharmacological activators of these two enzymes, makes them attractive targets for possible "exercise mimetics". This review will highlight the therapeutic potential of such putative "exercise mimetics", as well as the implications of using direct PGC-1 $\alpha$ activation as a treatment for metabolic and muscle-related disorders.

\section{AMPK / AICAR}

AMPK is activated in skeletal muscle during exercise and mediates both acute and long term metabolic adaptations to cope with the increased energy demand. Interestingly, in obese and type 2 diabetic individuals, activation of skeletal muscle AMPK during exercise is blunted [15]. This reduced activity of AMPK was also present in obese mice, and was further associated with reduced exercise capacity and diminished glucose uptake into skeletal muscle cells [16]. In this context, it therefore would be useful to enhance the positive effects of endurance training in obese patients by pharmacological activation of AMPK in skeletal muscle. One potential candidate for this is AICAR (5Aminoimidazole-4-carboxamide ribonucleotide), an AMP analog that can activate AMPK in vivo [13]. AICAR administration has been shown to reduce obesity and improve glucose homeostasis in mice [17] and to increase glucose uptake in skeletal muscle of both healthy and T2DM patients [18], which reflect the potential therapeutic use of AICAR as an "exercise mimetic". Importantly, AICAR can boost PGC-1 $\alpha$ transcription and posttranslational modifications in skeletal muscle [19]. Thereby, AICAR enhances endurance performance and energy expenditure through increased transcription of mitochondrial and metabolic genes in sedentary mice [20]. PGC-1 $\alpha$ played a central role in mediating these "exercise mimetic" effects of AMPK activation, since at least some genes activated by AICAR administration have been demonstrated to be dependent on PGC-1 $\alpha$ [21]. Due to the beneficial effects on skeletal muscle function and metabolism, AICAR has also been studied in the context of muscular dystrophies and muscle wasting. In angiotensin II-mediated muscle wasting in mice, daily AICAR administration could normalize AMPK activity and thereby reverse the loss of skeletal muscle mass [22]. In contrast, AICAR could not attenuate the decrease in muscle mass induced by denervation in mice, even though PGC-1 $\alpha$ expression and mitochondrial protein content in atrophic muscles were normalized [23]. These divergent effects of AICAR on attenuation of skeletal muscle wasting could be dependent on the different etiologies of these two atrophic mouse models, and would represent a variable therapeutic effect of AICAR depending on the nature of the muscle wasting disorder. In several recent papers, AICAR has been used to treat mdx mice, a model for Duchenne muscular dystrophy (DMD). In this model, AICAR administration improved skeletal muscle function through pleiotropic effects including increased mitochondrial activity, reduced inflammation 
and increased mitophagy [24-26]. Furthermore, AICAR treatment in combination with the peroxisome proliferator-activated receptor $\delta$ (PPARס) agonist GW1516 was shown to potentiate AMPK-activity during concomitant exercise in $\mathrm{mdx}$ mice [27], and could prove effective in enhancing beneficial effects of exercise in DMD patients.

Thus, in summary, AMPK activation in skeletal muscle by AICAR can lead to several beneficial effects in obese and diabetic mouse models. In atrophic and dystrophic mouse models, AICAR can improve muscle function and mitochondrial activity, although attenuation of skeletal muscle loss could not be demonstrated in all atrophic mouse models.

\section{SIRT1 / resveratrol}

Resveratrol (RSV) is a naturally occurring antioxidant that activates SIRT1 in vivo, and thereby promotes PGC-1 $\alpha$ deacetylation [28,29]. In obese sedentary mice, RSV improves exercise performance, ameliorates metabolic dysfunction and extends lifespan [28,29]. RSV could also boost mitochondrial adaptations in skeletal muscle in response to exercise [30,31], and some of these additive effects of RSV and exercise were further demonstrated to be dependent on skeletal muscle SIRT1 [31]. These findings have made RSV an interesting candidate for treating obesity and T2DM also in human subjects. In a recent clinical study, oral RSV administration did not affect either body weight or insulin sensitivity in obese patients [32], although in a similar study, RSV supplementation mediated mild metabolic adaptations combined with an increased transcription of mitochondrial genes in skeletal muscle [33]. In T2DM patients on the other hand, RSV administration has shown potential to improve glycemic control and insulin sensitivity [34,35]. RSV has also been investigated as a potential treatment for muscle wasting. In a rat model of mechanical unloading, RSV administration could prevent the decline in skeletal muscle oxidative capacity [36] , and attenuate the loss of skeletal muscle strength induced by muscle disuse [36,37]. RSV was also found to attenuate skeletal muscle wasting in a mouse model for cancer-associated cachexia, an effect that was associated with a reduced nuclear factor kappa-light-chain-enhancer of activated B cells (NFKB) activation and a reduction in muscle RING-finger protein 1 (MuRF1) expression [38]. The protective effects of RSV in these mouse models of skeletal muscle atrophy could be attributed to the activation of PGC-1 $\alpha$, since this co-activator has been shown to reduce NFKB driven transcription and attenuate activation of atrophic pathways in skeletal muscle [23,39]. Similar to AICAR, RSV has also been studied in the context of muscle dystrophy. In dystrophic mdx mice, RSV could attenuate the decline in skeletal muscle mass [40] and fatigue resistance [41], but could not ameliorate the ongoing inflammation or muscle injury [40]. In contrast, a different study in young mice could demonstrate an 
ameliorating effect of RSV treatment also on the infiltration of inflammatory cells into dystrophic muscle [42].

Thus, RSV administration has shown therapeutic potential for both metabolic and muscle wasting disorders in mice, while in human obese subjects, the beneficial effects of oral RSV administration are so far less promising.

\section{PGC-1 $\alpha-a$ central regulator in exercise}

Physical exercise induces a wide range of signaling pathways, all contributing to the metabolic and functional adaptations of skeletal muscle. The beneficial effects of activating a single one of these pathways through pharmacological modulation of either AMPK or SIRT1 have been demonstrated in several rodent disease models. This approach to designing "exercise mimetics" has yielded encouraging results, but considering the complex response to exercise in skeletal muscle, these compounds could only be considered as partial "exercise mimetics". A more complete "exercise mimetic" would instead directly target a central effector that could mediate both metabolic and functional adaptations to exercise in the skeletal muscle, and one such potential target would be PGC-1 $1 \alpha$.

Skeletal muscle PGC-1 $\alpha$ drives several gene programs that are important for muscle adaptation to endurance exercise. This is evident in mice overexpressing PGC-1 $\alpha$ specifically in skeletal muscle, which demonstrate a shift towards a slow-twitch oxidative phenotype, characterized by an increased mitochondrial biogenesis and higher fatigue resistance [43]. These changes are consistent with what has been observed in humans during acute endurance exercise, where an increase in PGC-1 $\alpha$ was associated with a slow type muscle fiber phenotype [10]. PGC-1 $\alpha$ can also regulate other processes that are important for exercise adaptation, such as muscle fatigue resistance and force generation [44], adaptation of the neuromuscular junction [45], angiogenesis [46], as well as glycogen storage [47]. PGC-1 $\alpha$ overexpression in skeletal muscle of mice resulted in an increased endurance capacity combined with a higher utilization of lipids as energy substrate during exercise [48]. The opposite was seen in mice carrying a skeletal muscle-specific knockout of PGC-1 $\alpha$, where exercise performance was reduced [49]. Mice deficient for skeletal muscle PGC-1 $\alpha$ also displayed increased muscle damage after an acute exercise bout [49], demonstrating an important role for PGC-1 $\alpha$ in maintaining muscle integrity during exercise. PGC-1 $\alpha$ acts as a convergence point for many signaling events taking place in a contracting skeletal muscle, and drives a pleiotropic transcriptional response resulting in improved muscle metabolism and endurance capacity. This makes modulation of PGC-1 $\alpha$ an interesting target for the treatment of metabolic and muscle related diseases. 
PGC-1 $\alpha$ - a potential target for "exercise mimetics"

An important factor in the prevention and treatment of obesity is to increase energy expenditure. Importantly, resting energy expenditure is increased up to 48 hours after an acute exercise bout in humans [50]. Irisin, a myokine released from skeletal muscle during exercise is an obvious candidate to mediate this effect, since it has been shown to increase whole body energy expenditure through enhanced mitochondrial uncoupling in white adipose tissue [51]. PGC-1 $\alpha$ regulates the transcription of irisin in skeletal muscle, and in PGC-1 $\alpha$ muscle knockout mice, circulating plasma levels of this myokine were accordingly reduced [51]. Interestingly, in contrast to the increased resting whole body energy expenditure seen in mice with an ectopic overexpression of irisin in liver [51], no positive effect on this parameter could be demonstrated in chow fed mice overexpressing PGC-1 $\alpha$ in skeletal muscle $[48,52]$. These results could indicate a minor role for skeletal muscle PGC-1 $\alpha$ in the regulation of energy expenditure through irisin during physical inactivity, while still being able to modulate energy expenditure during exercise. This suggestion is further corroborated by the increased peak oxygen consumption seen in PGC-1 $\alpha$ transgenic mice during acute exercise [48].

In mice fed a high fat diet, increased skeletal muscle PGC-1 $\alpha$ was not followed by increased resting energy expenditure, altered weight gain or improved glucose and insulin levels [52]. Surprisingly, overexpression of PGC-1 $\alpha$ in muscle was instead even found to have a detrimental effect on insulin stimulated glucose uptake in high fat diet fed sedentary mice [52]. This increased insulin resistance was attributed to an increased uptake of fatty acids into muscle, which exceeded the capacity of mitochondrial lipid oxidation, and that combined with elevated de novo lipogenesis, resulted in increased intramuscular lipid storage and a concomitant reduction in skeletal muscle insulin sensitivity [52]. Importantly however, when these mice received a continuous exercise intervention during the high fat feeding, these detrimental effects were reversed, and muscle glucose uptake as well as whole body glucose homeostasis was improved [53], demonstrating a synergistic effect of physical exercise and PGC-1 $\alpha$ overexpression on glucose homeostasis. By increasing PGC-1 $\alpha$ in skeletal muscle, the metabolic flexibility in the muscle is increased, mimicking what has been demonstrated during physical exercise. These changes include an up-regulation of mitochondrial fat oxidation [54], but also shunting of glucose away from oxidation, towards glycogen synthesis [47] and lipogenesis [55]. Thus, if PGC-1 $\alpha$ activity was increased in skeletal muscle of sedentary individuals, this could potentially lead to an uncoupling of the anabolic re-fueling processes induced by exercise from the increase in energy expenditure during muscle contraction. In a sedentary obese state where circulating lipid levels are already increased, activation of PGC-1 $\alpha$ might result in 
exacerbated intramuscular lipid accumulation and peripheral insulin resistance. These results strongly suggest that using PGC-1 $\alpha$ as an "exercise mimetic" for the treatment of obesity and T2DM in the absence of actual physical activity could potentially worsen the metabolic dysfunction in skeletal muscle as well as at a whole body level.

However, activation of PGC-1 $\alpha$ might still prove to be a valid therapeutic option in the context of metabolic dysfunctions that are not primarily associated with increased obesity. One such example is during aging, where overexpression PGC-1 $\alpha$ in skeletal muscle of mice was shown to ameliorate aging-associated insulin resistance and improve the metabolic flexibility of aged skeletal muscles [56].

In aged mice, increased PGC-1 $\alpha$ expression also improved muscle function and endurance capacity in conjunction with reduced sarcopenia [56]. A similar protective role of PGC-1 $\alpha$ in skeletal muscle atrophy was also seen in fasting- and denervation-induced atrophy, where PGC-1 $\alpha$ overexpression could rescue oxidative capacity and reduce the induction of a transcriptional atrophic response in the muscle [39]. This effect of PGC-1 $\alpha$ was proposed to occur through inhibition of forkhead box 03 (FoxO3) and NFKB driven transcription of atrophy-specific ubiquitin ligases, leading to a reduction in proteolysis [23]. The ability of PGC-1 $\alpha$ to inhibit NFkB driven transcription has also recently been demonstrated for expression of pro-inflammatory cytokines in skeletal muscle [57]. In mice suffering from cancer-induced cachexia, PGC-1 $\alpha$ overexpression up-regulated mitochondrial biogenesis, but did not prevent the reduction in body weight and muscle mass associated with the disease [58]. Interestingly, the authors could also show that increased levels of PGC-1 $\alpha$ in skeletal muscle caused an increase in tumor size, an effect that was speculated to occur through growth-promoting myokines and could potentially limit the potential therapeutic use of PGC-1 $\alpha$ activation in this context [58]. In dystrophic mdx-mice on the other hand, transgenic PGC-1 $1 \alpha$ overexpression reduced muscle damage and improved muscle function [45]. These effects were also demonstrated in mice subjected to PGC-1 $1 \alpha$ gene transfer into dystrophic muscles, resulting in a fast-to-slow fiber type switch, increased expression of utrophin and increased satellite cell activation [41,59]. Increased muscle PGC-1 $\alpha$ also demonstrated a therapeutic potential in a mouse model for amyotrophic lateral sclerosis (ALS), where it could improve muscle function and endurance capacity of the mice even in the later stages of the disease, unfortunately in the absence of a positive effect on lifespan of the mice [60].

These results demonstrate a strong potential for PGC-1 $\alpha$ to attenuate muscle wasting in several etiologically distinct disease states, and furthermore to improve both the metabolic phenotype and function of the affected muscles. 


\section{Conclusions}

The potential of using AICAR and RSV to mimic exercise through activation of exercise-induced pathways in the sedentary skeletal muscle have shown therapeutic beneficial effects in both metabolic and muscle-related diseases (Table 1). Since it is hard to mimic all the metabolic, neuronal as well as mechanical stimuli that occur during an actual muscle contraction, these "exercise mimetic" compounds could instead be administered in combination with physical activity to enhance the therapeutic potential of exercise. A direct effect of such an "exercise enhancer" would be to boost the activation of certain pathways during exercise, and thereby increase the beneficial adaptations to exercise in the muscle, a method that has proven to be successful for both RSV treatment [30,31] and PGC-1 $\alpha$ activation [53]. On the other hand, in conditions where skeletal muscle dysfunction is a central part of the disease etiology, administration of an "exercise enhancer" to these patients could increase mobility and the ability to perform physical activities, and thereby improve life quality. The fact that PGC-1 $\alpha$ can activate gene programs associated with both mitochondrial and functional adaptations in skeletal muscle, makes it an important potential candidate for treatment of diseases where reduced muscle function is a main part of the etiology. In line with this, increased levels of PGC-1 $\alpha$ in skeletal muscle have been shown to ameliorate the decline in muscle function in mouse models associated with muscle-wasting diseases such as DMD [45] and ALS [60], as well as in aging [56] (Table 1). On the other hand, in metabolic diseases such as obesity and T2DM, PGC-1 $\alpha$ activation could instead prove to be detrimental, since it activates lipid refueling in skeletal muscle without a concomitant increase in energy expenditure, which could potentially exacerbate insulin resistance.

Therefore, activation of PGC-1 $\alpha$ in skeletal muscle could represent a potential way to improve muscle function in atrophic diseases, but could on the other hand be less suitable as a treatment for obesity in the absence of actual physical exercise. Finally, it is important to note that the experimental "exercise mimetics" AICAR, RSV and PPARס ligands [20] have several potential drawbacks that hinder broad application in humans, such as poor oral bioavailability and unwanted side-effects that could complicate chronic treatment [61]. Furthermore, it is debatable whether real "exercise mimetics" can even be developed [62]. Therefore, until such therapeutically deployable compounds are found, bona fide physical activity remains a cheap, yet effective intervention of choice for many diseases. 


\section{Acknowledgments}

Research in our laboratory is supported by the Swiss National Science Foundation, the Muscular Dystrophy Association USA (MDA), the SwissLife 'Jubiläumsstiftung für Volksgesundheit und medizinische Forschung', the Swiss Society for Research on Muscle Diseases (SSEM), the Swiss Diabetes Association, the Roche Research Foundation, the United Mitochondrial Disease Foundation (UMDF), the Association Française contre les Myopathies (AFM), the Neuromuscular Research Association Basel (NeRAB), the Gebert-Rüf Foundation "Rare Diseases" Program, the University of Basel and the Biozentrum.

Conflict of interest: The authors have no conflict of interest to declare. 


\section{References}

1 Handschin, C. and Spiegelman, B.M. (2008) The role of exercise and PGC1alpha in inflammation and chronic disease. Nature 454 (7203), 463-469

2 Hollmann, W. et al. (2007) Physical activity and the elderly. European journal of cardiovascular prevention and rehabilitation : official journal of the European Society of Cardiology, Working Groups on Epidemiology \& Prevention and Cardiac Rehabilitation and Exercise Physiology 14 (6), 730-739

3 Strasser, B. (2012) Physical activity in obesity and metabolic syndrome. Annals of the New York Academy of Sciences

4 Maddocks, M. et al. (2012) Therapeutic exercise in cancer cachexia. Critical reviews in oncogenesis 17 (3), 285-292

$5 \quad$ Markert, C.D. et al. (2011) Exercise and Duchenne muscular dystrophy: toward evidencebased exercise prescription. Muscle \& nerve 43 (4), 464-478

6 Carey, A.L. and Kingwell, B.A. (2009) Novel pharmacological approaches to combat obesity and insulin resistance: targeting skeletal muscle with 'exercise mimetics'. Diabetologia 52 (10), 2015-2026

7 Egan, B. and Zierath, J.R. (2013) Exercise metabolism and the molecular regulation of skeletal muscle adaptation. Cell metabolism 17 (2), 162-184

8 Catoire, M. et al. (2012) Pronounced effects of acute endurance exercise on gene expression in resting and exercising human skeletal muscle. PloS one 7 (11), e51066

9 Lira, V.A. et al. (2010) PGC-1alpha regulation by exercise training and its influences on muscle function and insulin sensitivity. American journal of physiology. Endocrinology and metabolism 299 (2), E145-161 Russell, A.P. et al. (2003) Endurance training in humans leads to fiber type-specific increases in levels of peroxisome proliferator-activated receptor-gamma coactivator- 1 and peroxisome proliferator-activated receptor-alpha in skeletal muscle. Diabetes 52 (12), 2874-2881

11 Ruderman, N.B. et al. (2010) AMPK and SIRT1: a long-standing partnership? American journal of physiology. Endocrinology and metabolism 298 (4), E751-760

12 Hardie, D.G. et al. (2012) AMPK: a nutrient and energy sensor that maintains energy homeostasis. Nature reviews. Molecular cell biology 13 (4), 251-262

13 McGee, S.L. and Hargreaves, M. (2010) AMPK-mediated regulation of transcription in skeletal muscle. Clinical science $118(8)$, 507-518

14 White, A.T. and Schenk, S. (2012) NAD(+)/NADH and skeletal muscle mitochondrial adaptations to exercise. American journal of physiology. Endocrinology and metabolism 303 (3), E308-321

15 Sriwijitkamol, A. et al. (2007) Effect of acute exercise on AMPK signaling in skeletal muscle of subjects with type 2 diabetes: a time-course and dose-response study. Diabetes 56 (3), 836848

16 Lee-Young, R.S. et al. (2011) Obesity impairs skeletal muscle AMPK signaling during exercise: role of AMPKalpha2 in the regulation of exercise capacity in vivo. International journal of obesity 35 (7), 982-989

17 Giri, S. et al. (2006) AICAR inhibits adipocyte differentiation in 3T3L1 and restores metabolic alterations in diet-induced obesity mice model. Nutrition \& metabolism 3, 31

18 Babraj, J.A. et al. (2009) Blunting of AICAR-induced human skeletal muscle glucose uptake in type 2 diabetes is dependent on age rather than diabetic status. American journal of physiology. Endocrinology and metabolism 296 (5), E1042-1048

19 Tadaishi, M. et al. (2011) Effect of exercise intensity and AICAR on isoform-specific expressions of murine skeletal muscle PGC-1alpha mRNA: a role of beta(2)-adrenergic receptor activation. American journal of physiology. Endocrinology and metabolism 300 (2), E341-349 
20 Narkar, V.A. et al. (2008) AMPK and PPARdelta agonists are exercise mimetics. Cell 134 (3), 405-415

21 Jager, S. et al. (2007) AMP-activated protein kinase (AMPK) action in skeletal muscle via direct phosphorylation of PGC-1alpha. Proceedings of the National Academy of Sciences of the United States of America 104 (29), 12017-12022

22 Tabony, A.M. et al. (2011) Angiotensin II upregulates protein phosphatase 2Calpha and inhibits AMP-activated protein kinase signaling and energy balance leading to skeletal muscle wasting. Hypertension 58 (4), 643-649

23 Brault, J.J. et al. (2010) Peroxisome proliferator-activated receptor gamma coactivator 1alpha or 1 beta overexpression inhibits muscle protein degradation, induction of ubiquitin ligases, and disuse atrophy. The Journal of biological chemistry 285 (25), 19460-19471

24 Ljubicic, V. et al. (2011) Chronic AMPK activation evokes the slow, oxidative myogenic program and triggers beneficial adaptations in $\mathrm{mdx}$ mouse skeletal muscle. Human molecular genetics 20 (17), 3478-3493

25 Jahnke, V.E. et al. (2012) Metabolic remodeling agents show beneficial effects in the dystrophin-deficient mdx mouse model. Skeletal muscle 2 (1), 16

26 Pauly, M. et al. (2012) AMPK activation stimulates autophagy and ameliorates muscular dystrophy in the mdx mouse diaphragm. The American journal of pathology 181 (2), 583-592

27 Bueno Junior, C.R. et al. (2012) Combined effect of AMPK/PPAR agonists and exercise training in $\mathrm{mdx}$ mice functional performance. PloS one 7 (9), e45699

28 Baur, J.A. et al. (2006) Resveratrol improves health and survival of mice on a high-calorie diet. Nature 444 (7117), 337-342

29 Lagouge, M. et al. (2006) Resveratrol improves mitochondrial function and protects against metabolic disease by activating SIRT1 and PGC-1alpha. Cell 127 (6), 1109-1122

30 Dolinsky, V.W. et al. (2012) Improvements in skeletal muscle strength and cardiac function induced by resveratrol during exercise training contribute to enhanced exercise performance in rats. The Journal of physiology 590 (Pt 11), 2783-2799

31 Menzies, K.J. et al. (2013) Sirtuin 1-mediated Effects of Exercise and Resveratrol on Mitochondrial Biogenesis. The Journal of biological chemistry 288 (10), 6968-6979

32 Poulsen, M.M. et al. (2013) High-dose resveratrol supplementation in obese men: an investigator-initiated, randomized, placebo-controlled clinical trial of substrate metabolism, insulin sensitivity, and body composition. Diabetes 62 (4), 1186-1195

33 Timmers, S. et al. (2011) Calorie restriction-like effects of 30 days of resveratrol supplementation on energy metabolism and metabolic profile in obese humans. Cell metabolism 14 (5), 612-622

34 Brasnyo, P. et al. (2011) Resveratrol improves insulin sensitivity, reduces oxidative stress and activates the Akt pathway in type 2 diabetic patients. The British journal of nutrition 106 (3), 383-389

35 Bhatt, J.K. et al. (2012) Resveratrol supplementation improves glycemic control in type 2 diabetes mellitus. Nutrition research 32 (7), 537-541

36 Momken, I. et al. (2011) Resveratrol prevents the wasting disorders of mechanical unloading by acting as a physical exercise mimetic in the rat. FASEB journal : official publication of the Federation of American Societies for Experimental Biology 25 (10), 3646-3660

37 Jackson, J.R. et al. (2010) Mediation of endogenous antioxidant enzymes and apoptotic signaling by resveratrol following muscle disuse in the gastrocnemius muscles of young and old rats. American journal of physiology. Regulatory, integrative and comparative physiology 299 (6), R1572-1581

38 Shadfar, S. et al. (2011) Oral resveratrol therapy inhibits cancer-induced skeletal muscle and cardiac atrophy in vivo. Nutrition and cancer 63 (5), 749-762

39 Sandri, M. et al. (2006) PGC-1alpha protects skeletal muscle from atrophy by suppressing FoxO3 action and atrophy-specific gene transcription. Proceedings of the National Academy of Sciences of the United States of America 103 (44), 16260-16265 
Hori, Y.S. et al. (2011) Resveratrol ameliorates muscular pathology in the dystrophic $\mathrm{mdx}$ mouse, a model for Duchenne muscular dystrophy. The Journal of pharmacology and experimental therapeutics 338 (3), 784-794

41 Selsby, J.T. et al. (2012) Rescue of dystrophic skeletal muscle by PGC-1alpha involves a fast to slow fiber type shift in the mdx mouse. PloS one 7 (1), e30063

42 Gordon, B.S. et al. (2013) Resveratrol decreases inflammation and increases utrophin gene expression in the mdx mouse model of duchenne muscular dystrophy. Clinical nutrition 32 (1), 104-111

43 Lin, J. et al. (2002) Transcriptional co-activator PGC-1 alpha drives the formation of slowtwitch muscle fibres. Nature 418 (6899), 797-801

44 Summermatter, S. et al. (2012) Remodeling of calcium handling in skeletal muscle through PGC-1alpha: impact on force, fatigability, and fiber type. American journal of physiology. Cell physiology 302 (1), C88-99

45 Handschin, C. et al. (2007) PGC-1alpha regulates the neuromuscular junction program and ameliorates Duchenne muscular dystrophy. Genes \& development 21 (7), 770-783

46 Arany, Z. et al. (2008) HIF-independent regulation of VEGF and angiogenesis by the transcriptional coactivator PGC-1alpha. Nature 451 (7181), 1008-1012

47 Wende, A.R. et al. (2007) A role for the transcriptional coactivator PGC-1alpha in muscle refueling. The Journal of biological chemistry 282 (50), 36642-36651

48 Calvo, J.A. et al. (2008) Muscle-specific expression of PPARgamma coactivator-1alpha improves exercise performance and increases peak oxygen uptake. Journal of applied physiology 104 (5), 1304-1312

49 Handschin, C. et al. (2007) Skeletal muscle fiber-type switching, exercise intolerance, and myopathy in PGC-1alpha muscle-specific knock-out animals. The Journal of biological chemistry 282 (41), 30014-30021

50 Jamurtas, A.Z. et al. (2004) The effects of a single bout of exercise on resting energy expenditure and respiratory exchange ratio. European journal of applied physiology 92 (4-5), 393-398

51 Bostrom, P. et al. (2012) A PGC1-alpha-dependent myokine that drives brown-fat-like development of white fat and thermogenesis. Nature 481 (7382), 463-468

52 Choi, C.S. et al. (2008) Paradoxical effects of increased expression of PGC-1alpha on muscle mitochondrial function and insulin-stimulated muscle glucose metabolism. Proceedings of the National Academy of Sciences of the United States of America 105 (50), 19926-19931

53 Summermatter, S. et al. (2013) PGC-1alpha improves glucose homeostasis in skeletal muscle in an activity-dependent manner. Diabetes 62 (1), 85-95

54 Summermatter, S. et al. (2011) Coordinated balancing of muscle oxidative metabolism through PGC-1alpha increases metabolic flexibility and preserves insulin sensitivity. Biochemical and biophysical research communications 408 (1), 180-185

55 Summermatter, S. et al. (2010) Peroxisome proliferator-activated receptor \{gamma\} coactivator 1 \{alpha\} (PGC-1\{alpha\}) promotes skeletal muscle lipid refueling in vivo by activating de novo lipogenesis and the pentose phosphate pathway. The Journal of biological chemistry 285 (43), 32793-32800

56 Wenz, T. et al. (2009) Increased muscle PGC-1alpha expression protects from sarcopenia and metabolic disease during aging. Proceedings of the National Academy of Sciences of the United States of America 106 (48), 20405-20410

57 Eisele, P.S. et al. (2013) The peroxisome proliferator-activated receptor gamma coactivator 1alpha/beta (PGC-1) coactivators repress the transcriptional activity of NF-kappaB in skeletal muscle cells. The Journal of biological chemistry 288 (4), 2246-2260

58 Wang, X. et al. (2012) Increase in muscle mitochondrial biogenesis does not prevent muscle loss but increased tumor size in a mouse model of acute cancer-induced cachexia. PloS one 7 (3), e33426 
59 Hollinger, K. et al. (2013) Rescue of dystrophic skeletal muscle by PGC-1alpha involves restored expression of dystrophin associated protein complex components and satellite cell signaling. American journal of physiology. Regulatory, integrative and comparative physiology

60 Da Cruz, S. et al. (2012) Elevated PGC-1alpha activity sustains mitochondrial biogenesis and muscle function without extending survival in a mouse model of inherited ALS. Cell metabolism 15 (5), 778-786

61 Hawley, J.A. and Holloszy, J.O. (2009) Exercise: it's the real thing! Nutrition Reviews 67 (3), 172-178

62 Booth, F.W. and Laye, M.J. (2009) Lack of adequate appreciation of physical exercise's complexities can pre-empt appropriate design and interpretation in scientific discovery. Journal of Physiology-London 587 (23), 5527-5540

63 Jackson, J.R. et al. (2011) Long-term supplementation with resveratrol alleviates oxidative stress but does not attenuate sarcopenia in aged mice. The Journals of Gerontology. Series A, Biological sciences and medical sciences 66 (7), 751-764

64 Summermatter, S. et al. (2013) Skeletal muscle PGC-1 $\alpha$ controls whole-body lactate homeostasis through estrogen-related receptor $\alpha$-dependent activation of LDH B and repression of LDH A. Proceedings of the National Academy of Sciences of the United States of America 110 (21), 8738-8743 


\section{Figure Legend}

\section{Figure 1. Schematic representation of the processes involved in AMPK- and SIRT1-mediated}

activation of PGC-1 $\alpha$.

During physical exercise AMPK and SIRT1 are activated in skeletal muscle. Cross-talk between these two proteins occurs through SIRT1-mediated deacetylation of the AMPK-kinase LKB1, and through the ability of AMPK to raise intracellular NAD+ levels to activate SIRT1 [11]. Coordinated activation of AMPK and SIRT1 leads to activation of PGC-1 $\alpha$ through AMPK-mediated phosphorylation and subsequent deacetylation by SIRT1. PGC-1 $\alpha$ then interacts with several transcription factors, to mediate the induction of gene programs important for skeletal muscle adaptation to exercise (see box), through either direct or indirect transcriptional modulation. AICAR and resveratrol are pharmacological compounds that can activate AMPK and SIRT1 respectively, and thereby increase activation of PGC-1 $\alpha$ in skeletal muscle. Due to their ability to activate signaling pathways normally induced during physical exercise, AICAR and resveratrol have been termed "exercise mimetics" [61].

Abbreviations: TF, transcription factor; LKB1, liver kinase B1; NAD+, Nicotinamide adenine dinucleotide; PGC-1 $\alpha$, peroxisome proliferator-activated receptor $\gamma$ coactivator 1- $\alpha$; NMJ, neuromuscular junction; Esrra, estrogen-related receptor $\alpha$; Tfam, transcription factor $A$, mitochondrial; Cycs, cytochrome complex; Vegf, vascular endothelial growth factor; Angpt2, angiopoietin-2; Pdgfb, platelet-derived growth factor subunit B; $\mathrm{AChR} \alpha$, acetylcholine receptor $\alpha$; Utrn, utrophin; Gabpa, GA-binding protein alpha chain; Fasn, fatty acid synthase; Dgat, diglyceride acyltransferase; Gpam, mitochondrial glycerol-3-phosphate acyltransferase; Atp2a1, sarco/endoplasmic reticulum Ca2+-ATPase 1; Casq1, calsequestrin 1; Casq2, calsequestrin 2; Ldha, lactate dehydrogenase $a$; Ldhb, lactate dehydrogenase b; Slc16a1, monocarboxylate transporter 1. Denotations: (P), phosphorylation; (Ac), acetylation; $\uparrow$, up-regulation; $\downarrow$, down-regulation. 


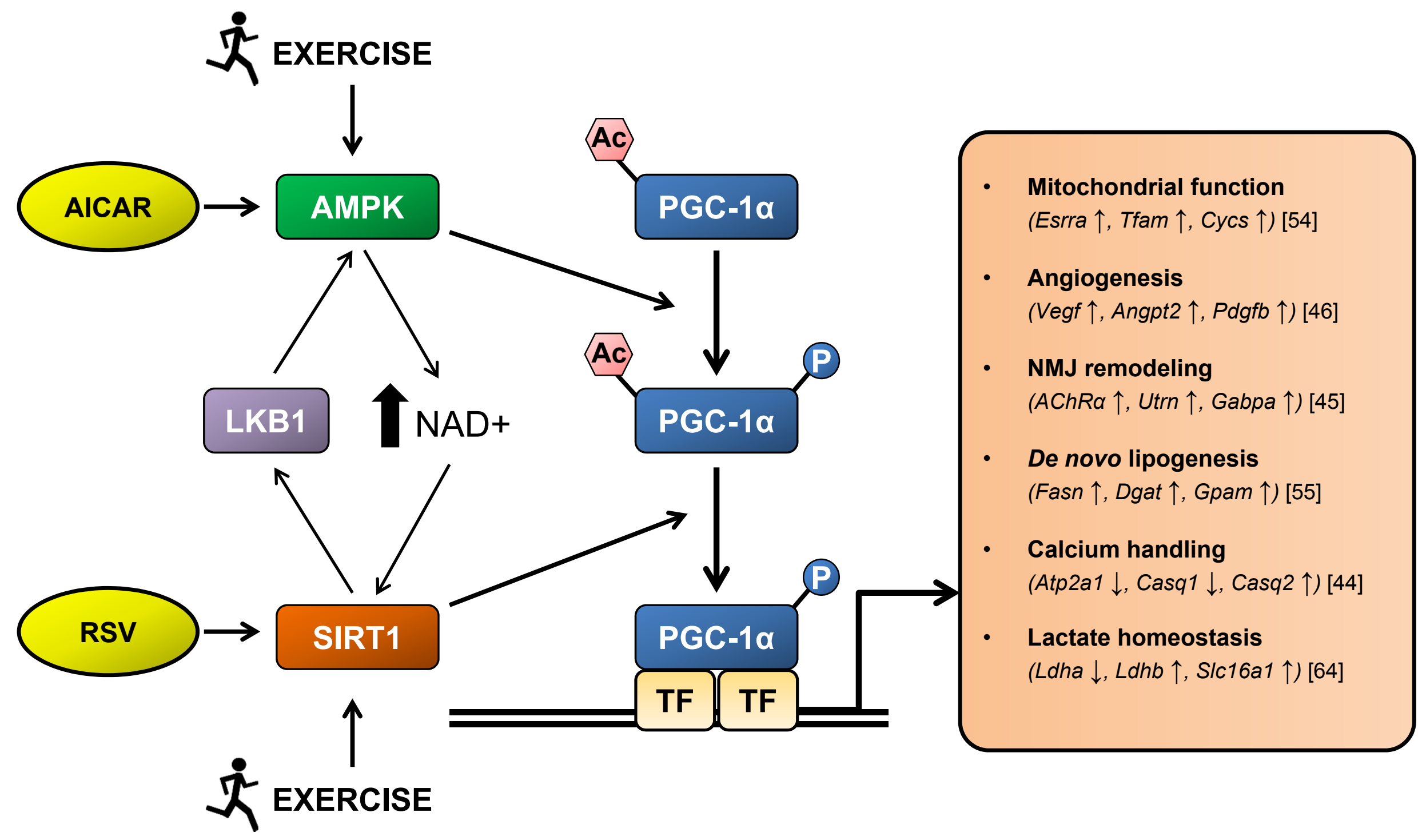


Table 1. Effects of AICAR, resveratrol and PGC-1a overexpression on metabolism and muscle function in rodent disease models.

Disease models

Obesity / Type 2 diabetes

Aging

Not determined

Muscle wasting $\downarrow$ (22)
Obesity $\downarrow$

Obesity $\downarrow$ (17)

Insulin sensitivity $\uparrow$ (17)

\section{Resveratrol}

nsulin sensitivity $\uparrow(28,29)$

Exercise performance $\uparrow$ (28)

Life span $\uparrow$ (29)
PGC-1a overexpression

Intramuscular lipid storage $\uparrow$ (52) Insulin sensitivity $\downarrow$ (52)

\section{in combination with exercise}

Glucose homeostasis $\uparrow$ (53)

Insulin sensitivity $\uparrow$

Insulin sensitivity $\uparrow$ (56)

Exercise performance $\uparrow$ (56)

Muscle wasting $\downarrow$ (56)
Angiotensin II induced atrophy

Disuse/denervation induced atrophy

Cancer-associated cachexia

Not determined
Muscle wasting $\downarrow$ (38)
Oxidative capacity $\uparrow$ (36)

Muscle force $\uparrow(36,37)$
Oxidative capacity $\uparrow$ (39)

Muscle wasting $\downarrow$ (39)
Mitochondrial activity $\uparrow$ (58)

Muscle wasting $\leftrightarrow$ (58)

Tumor size $\uparrow$ (58)

\section{Duchenne muscular dystrophy (DMD)}

Mitochondrial proteins $\uparrow$ (24-25)

Muscle weight $\uparrow$ (25)

Inflammation $\downarrow$ (25)
Muscle wasting $\downarrow$ (40)
Inflammation $\downarrow$ (42)
Muscle function $\uparrow(41)$

Not determined
Muscle function $\uparrow(60)$

Exercise performance $\uparrow$ (60)

Life span $\leftrightarrow(60)$

$$
\text { Life span } \leftrightarrow(60)
$$

Muscle function $\uparrow(41,45,59)$

Muscle damage $\downarrow(41,45,59)$

\section{Amyotrophic lateral sclerosis (ALS)}

Not determined

Abbreviations: PGC-1 $\alpha$, peroxisome proliferator-activated receptor $\gamma$ co-activator $1 \alpha$

Denotations: $(\uparrow)$ indicates an increase and $(\downarrow)$ indicates a decrease of the described process/phenotype in treated/transgenic mice compared to untreated/wild-type mice. $(\leftrightarrow)$ indicates no difference between treated/transgenic mice and untreated/wildtype mice. 